xviii

\title{
The theorem of Apollonius by dissection.
}

The figure shews the dissection in the form

$$
\left(A B^{2}-A D^{2}\right)+\left(A C^{2}-A D^{2}\right)=2 B D^{2} .
$$

Let $m, x$ denote $A D, D E$, where $E$ is the projection of $A$ on $B C$.

Quadrilateral $l$ is constructed with sides $\frac{1}{2}(c+x), \frac{1}{2}(c-x)$, $\frac{1}{2}\left(m+\frac{1}{2} a+x\right), \frac{1}{2}\left(m-\frac{1}{2} a-x\right) ;$ quadrilateral 6 with sides $\frac{1}{2}(b-x)$, $\frac{1}{2}(b+x), \frac{1}{2}\left(m-\frac{1}{2} a-x\right), \frac{1}{2}\left(m+\frac{1}{2} a-x\right)$. The square on $A B$ is then dissected into four quadrilaterals equal to 1 , plus the shaded square $\left(A D^{2}\right)$; similarly for the square on $A C$. These quadrilaterals are reassembled in the squares on $B E, E C$ respectively with the rightangled corners in the reversed positions, so as to enclose squares each equal to $D E^{2}$. Finally two quadrilaterals are subdivided $(4,11)$ and $(3,5,10,12)$ to fit in as shewn in the figure.

To avoid difficulties of overlapping in this method of dissection the triangle should be drawn with $A D$ much smaller than either $A B$ or $A C$.

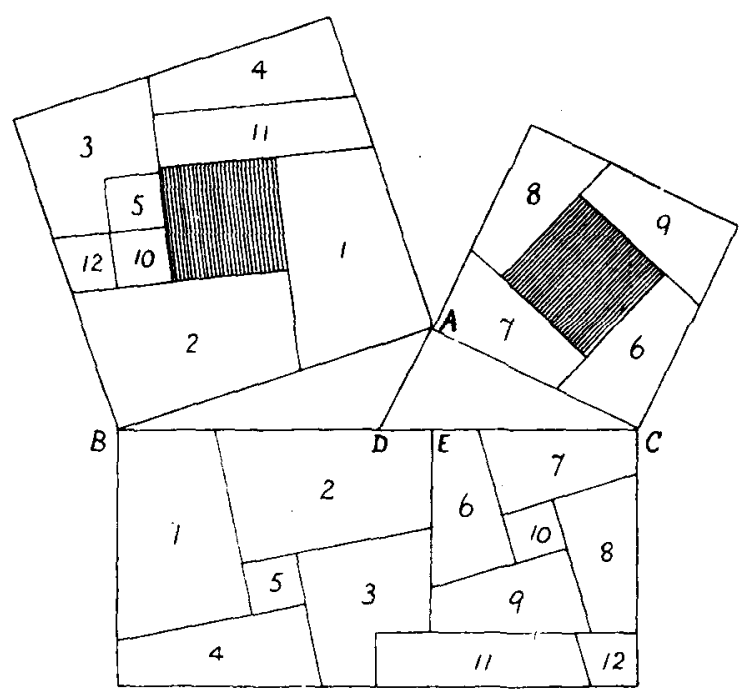

G. D. C. Stokes. 